\title{
Analytical Comparison between Microhematocrit and Automated Methods for Packed Cell Volume (PCV) Determination
}

\author{
Audu I. Stephen ${ }^{1 *}$, Simon T. Ubwa ${ }^{1}$, Ogbene G. Igbum ${ }^{1}$, Stephen S. Hati ${ }^{2}$, NwannadiI.Alex ${ }^{3}$ \\ ${ }^{1}$ Department of Chemistry, Benue State University, Makurdi, Nigeria \\ ${ }^{2} R R \& P$, Nisa Premier Hospital, Abuja, Nigeria \\ ${ }^{3}$ Department of Haematology, Benue State University Teaching Hospital, Makurdi, Nigeria
}

Received: $6^{\text {th }}$ June, 2017; Accepted: $26^{\text {th }}$ June, 2017; Published: $10^{\text {th }}$ July, 2017

*Corresponding author: Audu I. Stephen, Department of Chemistry, Benue State University, Makurdi, Nigeria.E-mail: rexaudu@gmail.com

\begin{abstract}
Analytical methods comparison for the determination of packed cell volume(PCV) are essential in clinical laboratory practice as it improves the quality of health care through accurate and reliable clinical decision making from diagnostic results of suitable alternatives. Comparison is necessary because each method is expected to serve as a quality control measure for the other. This study was done to assess the analytical performance between the Microhematocrit and automated methods for PCV determination. In this study carried out at the heamatology laboratory of the Benue State University Teaching Hospital, Makurdi, Nigeria, the Microhematocrit method determined by using the HC 702, (ApelCo. Ltd, Korea) was compared with the Automated hematology Analyzer method (KX-21N sysmex, USA) using paired data of blood samples analyzed respectively from 206 patients in the hospital. Data analysis was performed using Analyse-it ${ }^{\circledR}$ Version 4.6 method validation software. The results showed that similar overall mean values of PCV were obtained by both microhematocrit $(34.5 \pm 7.3 \%)$ and automated $(34.3 \pm 6.8 \%)$ methods. Result of t-test analysis was not statistically significant $(\mathrm{p}=0.135)$ between the overall measurements by both methods.
\end{abstract}

Pearson's analysis revealed a high correlation value $(r=0.917)$ between microhematocrit and Automated measurements of PCV. Passing-Bablok fit of the regression line provided the equation: Automated $=1.373+0.9692$ microhematocrit; and slope value (0.969, 95\% CI: 0.9400 to 1.008 ) supports the high correlation coefficient. The Bland-Altman plot of mean difference expresses high level of agreement between the microhematocrit and Automated measurements. The average error in evaluating PCV with microhematocrit compared with evaluation with Automated (calculated by Automated - microhematocrit) ranged between 16.2\% and $59.3 \%$ ( $95 \% \mathrm{CI}=0.9400 \%$ to $1.008 \%$ ). It can be concluded that despite the sophistication of present day auto analyzers, there is need to depend on manual techniques for primary calibration, and the study confirm that the microhematocrit readings are as reliable as the automated haematology analyzer.

Keywords: Microhematocrit; Packed cell Volume; Automated method; Anaemia

\section{Introduction}

The packed cell volume (PCV) test are normally done to diagnose or evaluate anaemia (decrease of red blood cells), polycythaemia (increased in red blood cells). Conditions that can lead to low PCV include, bleeding, kidney disease (a healthy kidney secretes a hormone erythropoietin which stimulates red blood cell production in the bone marrow) and heamolysis (where the red blood cells are being destroyed prematurely either due to attack by the body immune system or organ damage) [1]. Hematocrit is the percentage of blood that is comprised of red blood cell. This is often referred to as packed cell volume (PCV) or erythrocyte volume fraction. It is considered as an integral part of a person's complete blood count, along with hemoglobin concentration, white blood cell count and platelet counts [2,3]. The measurement of the packed cell volume (PCV) is useful in any hematologic workup and is a main tool in the quality control programs in the haematology laboratory [4]. Incorrectly reported microhematocrit result may bias clinical decision in follow up of patients, blood transfusion decision, and in diagnosis of hematologic diseases such as severe anemia. In spite of its significance it has received far less consideration in research from the standpoint of its reliability than have the measurements of hemoglobin or red cell counts [3,4].

In Nigeria, hematocrit is a common complete blood count parameter routinely used by clinicians. In most rural part of the country where the automated analysers are not available, the microhematocrit method is used to determine PCV in clients. Haematology autoanalyzers provide quick and accurate results in most situations. However, auto analyzers are prone to errors as platelet aggregates or hypolobulated neutrophil may give rise to false high PCV $[5,6]$.

\section{Materials and Methods}

\section{Study Setting and Design}

TThe study was carried out at the haematology laboratory of the Benue State University Teaching hospital, Makurdi, Nigeria. 
This prospective study was conducted from November 2015 to September 2016. A total of 206 patients were randomly sampled at the Benue State University Teaching Hospital, Makurdi during the study period. The study was approved by the Health Research and Ethics Committee of the Hospital.

\section{Determination of Packed Cell Volume (PCV)}

$2 \mathrm{mls}$ of blood samples were collected from the patients into bottles containing Ethylene di amine tetra acetic acid (EDTA) anticoagulant and was properly mixed using a blood mixer. PCV was determined by the microhematocrit by placing the centrifuge heparinized blood in a capillary tube (also known as microhematocrit tube), the capillary tube was filled to $75 \%$ of its length, sealed with plasticine and centrifuged in a micro haematocrit centrifuge at 10,000 RPM for five minutes, this separates the blood into layers. It is then placed on the HC 702 hematocrit and read [7,8]. The Microhematocrit centrifuge components include a motor that supplies power to the shaft and rotor centrifuge heads (carriers) that spin on the rotor and a lid latch or lid. centrifuge apply centrifugal force to separate suspended particles from a liquid or to separate liquids of different densities, the micro hemetocrit centrifuge is a special purpose version of a fixed head unit, quickly attains speed of 11,000 rpm and RCFs (relative centrifugal force) of up to15,000g to spin micro capillary tube samples [16]. The Automated haematology Analyzer used the counter principle, which blood is passed between two electrodes through an aperture so narrow that only one cell can pass through at a time. The change in impedence is proportional to cell volume resulting in a cell count and measure of volume [6]. PCV was determined directly by placing the blood sample in the Automated hematology Analyzer (KX-21N sysmex, USA). The sysmex hematology automated analyser can be run on its own, or connected to a blood film making and staining unit. Racks of blood go in on a tray on the right and come out on the left side. The racks hold ten $4.5 \mathrm{~mL}$ tubes and have a notch so they only go in one way. The sampled blood moves through a tube thin enough that cells pass by one at a time, characteristics about the cell are measured using lasers (fluorescent flow cytometry) or electrical impedence [6]. The PCV values of both methods were properly recorded, all blood samples were analysed within $6 \mathrm{hrs}$ after collection and samples were analysed the same time by both the Microhematocrit and the automated method.

\begin{tabular}{|l|c|c|}
\hline \multicolumn{3}{|c|}{ Table 1: PCV samples characteristics } \\
\hline \multicolumn{1}{|c|}{ Statistic\% } & Automated & Microhematort \\
\hline Minimum & 16.5 & 16 \\
\hline Maximum & 59.3 & 49 \\
\hline Median & 35.0 & 35.0 \\
\hline Mean & 34.5 & 34.3 \\
\hline Mean SE & 0.71 & 0.66 \\
\hline SD & 7.3 & 6.8 \\
\hline Variance & 52.9 & 46.1 \\
\hline Skewness & 0.2 & -0.4 \\
\hline Kurtosis & 1.26 & 0.02 \\
\hline
\end{tabular}

\section{Statistical analysis}

Data collected were collated on Microsoft Excel spread sheet and analysis was done using Analyse-it ${ }^{\circledR}$ Version 4.6, (Analyseit Leeds, UK) [9] A p-value of less than 0.05 (p < 0.05) was considered significant.The software supports the latest CLSI and industry-recognised protocols, enables the analyst to validate, verify and demonstrate analytical accuracy, precision, linearity, reference intervals, and diagnostic performance [9].

\section{Results}

A total of 206 subjects were sampled and 412 paired data were obtained and analysed in this study. The results showed that similar overall mean values of PCV were obtained by both microhematocrit (34.5 $\pm 7.3 \%)$ and Automated (34.3 $\pm 6.8 \%)$. Result of t-test analysis was not statistically significant ( $p>0.05$ ) between the overall measurements by both microhematocrit and the Automated heamatology analyzer.

Pearson's analysis revealed a high correlation value $(\mathrm{r}=0.917)$ between microhematocrit and Automated measurements of PCV. Passing-Bablok fit of the regression line provided the equation: Automated $=1.373+0.9692$ microhematocrit; and slope value (0.969, 95\% CI: 0.9400 to 1.008$)$ supports the high correlation coefficient (Figure 1).

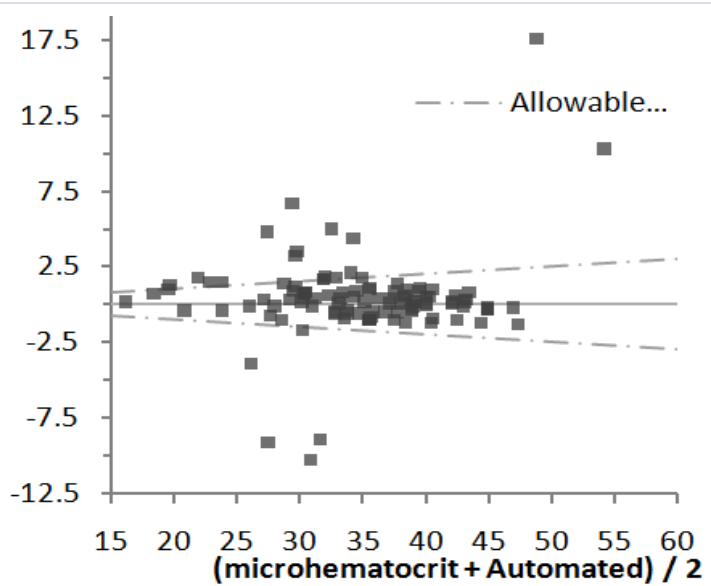

Figure 1: Pearson's correlation plot for PCV

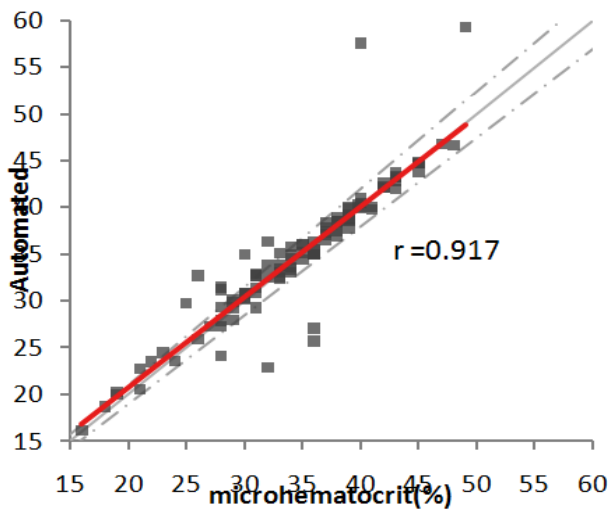

Figure 2: Bland-Altman plot comparing microhematocrit-Automated measurement 
The Bland-Altman plot of mean difference expresses high level of agreement between the microhematocrit and Automated measurements. The average error in evaluating PCV with microhematocrit compared with evaluation with Automated (calculated by Automated - microhematocrit) ranged between $16.2 \mathrm{~s} \%$ and $59.3 \%$ (95\% CI $=0.9400 \%$ to $1.008 \%$ ). A BlandAltman error plot illustrated that $<21 \%(99.0 \%)$ data points were positive; indicating that Automated measurements overestimated microhematocrit values (Figure 2).

\section{Discussion}

Results of this study indicated that similar overall mean values of PCV were obtained by both methods. A Bland-Altman error plot illustrated that $<21 \%(99.0 \%)$ data points were positive; indicating that Automated measurements over estimated microhematocrit values.

Automated method for the estimation of PCV is now mainly patronized in routine laboratory $[10,11]$, but that does not necessary mean that the Automated method has replaced the manual method of PCV determination, as so many other studies are in confirmation with this study which suggested that the microhematocrit readings are reliable as the automated hematology analyzer, even though the analyzer gives additional diagnostic information through the blood pictures hence, manual blood examination should always be used to validate the automated methods as suggested by Lantis, et al. [12]. However, the imprecision in the determination of PCV by the manual method Hematocrit may results in variations in red blood cell indices. The results of this study is in agreement with the earlier study reported by Ike S, et al [6] who reported that the automated hematology analyzer readings are as reliable as the microhematocrit. The pearson's analysis revealed a high correlation value $(\mathrm{r}=0.917)$ between the microhematocrit method and the Automated method, also the Bland-Altman plot of mean difference expresses a high agreement between the microhematocrit and Automated method.The average error in evaluating PCV with Hematocrit compared with evaluation with Automated (calculated by Automated - microhematocrit) ranged between $16.2 \%$ and $59.3 \%$ (95\% CI $=0.9400 \%$ to $1.008 \%$ ).

In contrast to this study which reported higher value of PCV in the Automated method and similar overall mean values between the both methods, study by Gebretsadkan, et al. [4] reported higher PCV value from the Manual hematocrit method compared to the Automated analyzer method [4]. Microhematocrit method is a gold standard method for hematocrit determination but it is associated with many problems that may lead to inaccurate and imprecise measurements. Spun hematocrit is $1 \%$ to $3 \%$ higher than the hematocrit from automated instrument due to plasma that is trapped in erythrocytes $[13,14]$.

The automated hematology autoanalyzer with complete blood count (CBC) results has replaced the traditional manual or individual assay methods for hematological parameters [15]. Hematology auto analyzers provide quick and accurate results in most situations, however, false results related either to platelet of other parameters from complete blood count may be observed in several instances. False low white blood cell WBC counts maybe observed because of agglutination in the presence of ethylene di amine tetra-acetic acid. Despite the sophistication of present day instruments, there is need to depend on manual techniques for primary calibration[6].The results of this study is in agreement with the study reported by Kakel, Sj, [15 ] in which higher PCV values were obtained from Coulter automated analyzer, eventhough there was no significant difference observed .

\section{Conclusion}

This study showed that despite the sophistication of present day Autoanalyzers, there is need to depend on manual techniques for primary calibration. This study revealed that the microhematocrit readings are as reliable as that of the automated haematology analyzer readings. The automated method cannot replace the manual for hematocrit determination though the results of both methods are complementary.

\section{Acknowledgement}

Authors acknowledge the support of the Head of Department of haematology and all the staffs of Haematology laboratory of Benue State University, Makurdi, Nigeria.

\section{References}

1. Association for chemical Biochemistry \& Laboratory Medicine, 2012: packed Cell Volume; heamatocrit. Availablefrom: http://labtestonline. org.Uk

2. Kathleen K (2007) The Clinical Laboratory Improvement Act (CLIA) and the Physician's Office Laboratory. Continuing Medical Education

3. CLSI. Procedure for Determining Packed Cell Volume by the Microhematocrit Method; Approved Standard. Third editon. CLSI, 940 West Valley Road, Suite 1400, Wayne, Pennsylvania 19087-1898, USA; 2000.

4. Gebretsadkan G, Tessema K, Ambachew H. The Comparison between Microhematocrit and Automated Methods for Hematocrit Determination ClinMed. Int J Blood Res Disord. 2015;2(1):1-3.

5. Zandecki M, Genevieve F, Gerald J, Gordon A. Spurious counts and spurious results on hematology analysers. International Journal of Laboratory hematology.2007; 29(1):21-41. doi: 10.1111/j.13652257.2006.00871

6.Samuel Ike, Thomas N, Ernest U, Imelda NN, Elvis Shu, Ezema I, et al. comparison of heamatological parameters determined by the Systnex KX-2IN automated heamatology analyzer and the manual counts BMC clinical pathology, Biomed central. 2010;10:3. doi: 10.1186/14726890-10-3

7. "Hematocrit. Encyclopedia of Surgery: A Guide for Patients and Caregiver.

8. CLSI. Procedure for Determining Packed Cell Volume by the Microhematocrit Method; Approved Standard. Third Edit. Vol. 20, USA; 2000 .

9. Analyse-it. Clinical and Analytical Method Validation Statistical 
Software for Microsoft Excel. Analyse-it Software, Ltd. Leeds, United Kingdom. Analyse-it v.4.6 2015.Available from: http://analyse-it. com/products/method-validation/.

10. i-STAT. Hematocrit Determination in the i-STAT ${ }^{\circledR}$ System and Comparison To Other Method Abbott Point of Care Inc. USA Art: 714261-00E. 2011.

11. Karem KK, Sabour N, Kulaif M. Comparison between manual procedure andautomated for determinant of WBCs and PCV in maternity and labor hospital in Karbala city. J Contemp Med Sci. 2016;2(7):93-95.

12. Lantis KL, Harris RJ, Davis G, Renner N, Finn WG. Elimination instrument - driven reflex manual deferential leucocyte counts optimization of manual blood smear review criteria in a high volume automated heamatology laboratory. Am J clinical pathology.

\section{3;119(5):656-662. doi: 10.1309/VH1K-MV8W-B7GB-7R14}

13. Pearson TC, Guthrie D L. Trapped Plasma in the Microhematocrit. AmJClinPathol. 1982;78(5):770-772

14. Gotch F,Torres L, Evans M, Keen M, Metzernerc, Westphal D. Comparison of Conductivity measured hematocrit to microhematocrit. 1991;ASAIO Trans 37(3):M138-139.

15. Kakel SJ.The evaluation of traditional and automatic Coulter Method in estimation of haematological parameters in adult rats.Beni-Suef University Journal of Basic and Applied Sciences. 2013;2(1):31-35.

16. WHO(2012).Centrifuges,Microhematocrit. Available from: http:// www.who.int/medical_devices/en/ 